\title{
Instability of the BCS type pairing in magnetic field due to the Rashba spin-orbit interaction and supercurrent generation by spin-twisting itinerant motion of electrons in BCS superconductors
}

\author{
Hiroyasu Koizumi \\ Division of Materials Science, University of Tsukuba,Tsukuba, Ibaraki 305-8573, Japan \\ Masashi Tachiki \\ Department of Physics, Tohoku University, Sendai, Miyagi 980-8578, Japan
}

(Dated: June 1, 2015)

\begin{abstract}
In a uniform magnetic field, the BCS type pairing is taken over by the pairing of spin-twisting itinerant motion states due to the Rashba spin-orbit interaction. It gives rise to stable eddy currents forced to flow by the single-valued requirement of the wave function. They are stabilized by the energy gap of the electron pairing and protected by topological winding numbers of the wave function for the spin-twisting itinerant motion. The supercurrent is generated by a collection of such eddy currents.
\end{abstract}

PACS numbers: 74.20.-z,74.20.Fg,74.72.-h 
The BCS theory succeeded in explaining the superconducting phase transition as an energy gap creation by the electron pairing (or the Cooper pair formation), and the transition temperature $T_{c}$ was identified as this energy gap formation temperature [1]. Supercurrent related phenomena have been well-described by the Ginzburg-Landau theory [2], and the connection between the BCS theory and the Ginzburg-Landau theory was provided by Gor'kov [3]; therefore, it had been believed that the BCS theory was a complete theory of superconductivity. The Josephson's prediction and its observation reinforced this belief [4].

The comfortable agreement between theory and experiment in superconductivity was upset by the discovery of the high temperature superconductivity in the cuprate [5]. The cuprate superconductivity shows marked deviations from the BCS theory. Especially, $T_{c}$ is not determined as the energy gap creation temperature, but argued to be given as the stabilization temperature of coherence-length-sized persistent loop currents [6]. In order to explain the cuprate superconductivity, a new supercurrent generation mechanism in which the coherence-length-sized persistent loop current is the protagonist of superconductivity has been proposed [7-11].

In the new supercurrent generation mechanism, the superconducting wave function is given in the following form

$$
\Psi\left(\mathbf{r}^{(1)}, \cdots, \mathbf{r}^{(N)}\right)=\Psi_{0}\left(\mathbf{r}^{(1)}, \cdots, \mathbf{r}^{(N)}\right) e^{-\frac{i}{2} \sum_{\alpha=1}^{N} \chi\left(\mathbf{r}^{(\alpha)}\right)}
$$

where $\mathbf{r}^{(j)}$ is the coordinate of the $j$ th electron and $N$ is the total number of electrons. $\Psi_{0}$ is a currentless multi-valued wave function, where the multi-valuedness arises from the spin-twisting of the itinerant electrons. The phase factor $e^{-\frac{i}{2} \sum_{\alpha=1}^{N} \chi\left(\mathbf{r}^{(\alpha)}\right)}$ arises to impose the single-valued requirement of the total wave function $[9,10]$. The single-valued requirement of the wave function is one of the postulates imposed by Schrödinger to evaluate hydrogen atomic spectrum [12]. It is known from the dynamical Jahn-Teller problem that if the same postulate is applied to the multi-component wave function system, a zero point circular motion becomes possible [13]. In the new supercurrent generation mechanism, the multicomponent is supplied by the spin-degree-of-freedom, and the zero point circular motion gives rise to a stable eddy current.

The ac Josephson frequency was re-derived using the new mechanism $[7,10]$. It explained the effect very well; besides, it revealed surprising facts: first, it was noted that the actual experiment employed a different boundary condition from the one assumed by Josephson 
[11]; in the actual experiment, a dc current is fed, while such a current feeding was not considered in the Josephson's prediction. Second, the predicted ac current across the Josephson junction, $J_{s}=J_{c} \sin \frac{2 e V}{\hbar} t$ ( $J_{c}$ is a critical current, $e$ is the absolute value of electron charge, $\hbar$ is Planck's constant divided by $2 \pi, V$ is a dc voltage across the junction, and $t$ is time), has never been observed [14-18], although text books state otherwise [19-21]. Third, the re-derivation performed using the actual experimental boundary condition indicates that the supercurrent carrier charge is $-e$ contrary to the Josephson's assumption $-2 e[7,10]$.

The above results suggest that the currently-accepted supercurrent generation mechanism may be wrong. Actually, when the BCS theory was first appeared, whether the BCS theory really explained the persistent current generation was questioned since the explanation of the Meissner effect was not done in a gauge invariant manner. This problem was taken up by many researchers and believed to be solved [22, 23]. However, the solution employs a formalism that does not conserve the particle number and utilizes phases that violates the superselection rule for charge [24, 25]; apparently, this solution is not applicable to isolated superconductors since the particle number is conserved in them. It is also noteworthy that Gor'kov's connection between the BCS theory and the Ginzburg-Landau theory requires the violation of the particle number conservation [3].

In the present work, we show that the new supercurrent generation mechanism by the spin-twisting itinerant motion of electrons is also applicable to the BCS superconductor. The spin-twisting itinerant motion of electrons required for the new supercurrent generation mechanism is realized by the Rashba spin-orbit interaction, which is effective even if the interaction is very weak.

Let us start with the BCS variational state vector. In the BCS ground state the pairing occurs between $(\mathbf{k}, \uparrow)$ and $(-\mathbf{k}, \downarrow)$, and the state vector is given by $\varphi_{\mathbf{k} \sigma}=\frac{1}{\sqrt{\mathcal{V}}} e^{i \mathbf{k} \cdot \mathbf{r}}|\sigma\rangle$, where $|\sigma\rangle$ is the spin state vector with $\sigma=\uparrow$ or $\downarrow, \mathcal{V}$ is the volume of the system, and $\mathbf{r}$ is the spatial coordinate. Using the creation operators $c_{\mathbf{k} \sigma}^{\dagger}$ for $\varphi_{\mathbf{k} \sigma}$, the BCS state vector is given by $|\mathrm{BCS}\rangle=\prod_{\mathbf{k}}\left(u_{\mathbf{k}}+v_{\mathbf{k}} c_{\mathbf{k} \uparrow}^{\dagger} c_{-\mathbf{k} \downarrow}^{\dagger}\right)|\operatorname{vac}\rangle$, where $u_{\mathbf{k}}$ and $v_{\mathbf{k}}$ are real variational parameters that satisfy $u_{\mathbf{k}}^{2}+v_{\mathbf{k}}^{2}=1$ [1]. This particle number non-fixed state vector facilitates calculations involving the electron pair-correlation that yields an energy gap in single-particle excitations. It is known that the particle number distribution has a sharp peak at the mean-value $N$, thus, the obtained wave function can be practically regarded as that for a state with $N$ particles. We take this view through out. 
In the following, we add the Rashba spin-orbit interaction, $H_{s o}=\lambda \mathbf{E}^{\mathrm{em}} \cdot(\boldsymbol{\sigma} \times \hat{\mathbf{p}})$ to the BCS theory, where $\lambda$ is the spin-orbit coupling parameter, $\mathbf{E}^{\mathrm{em}}$ is the electric field, $\boldsymbol{\sigma}$ is the vector of Pauli matrices, and $\hat{\mathbf{p}}$ is the momentum operator [26]. By taking the electric field in the $z$ direction, $\mathbf{E}^{\mathrm{em}}=\left(0,0, E_{\mathrm{em}}\right), H_{s o}$ is written as

$$
\begin{aligned}
H_{\text {so }}^{\mathrm{cyl}}= & \lambda E_{\mathrm{em}}\left[e^{-i \phi}\left(\frac{\partial}{\partial \rho}-\frac{i}{\rho} \frac{\partial}{\partial \phi}\right)|\uparrow\rangle\langle\downarrow|\right. \\
& \left.-e^{i \phi}\left(\frac{\partial}{\partial \rho}+\frac{i}{\rho} \frac{\partial}{\partial \phi}\right)|\downarrow\rangle\langle\uparrow|\right],
\end{aligned}
$$

where $\rho$ and $\phi$ are cylindrical coordinates related to the $x$ and $y$ coordinates as $x=\rho \cos \phi$ and $y=\rho \sin \phi$, respectively.

First, we examine a cylindrical symmetry system with a harmonic oscillator potential in the $x-y$ plane. By adding the harmonic oscillator potential, the zeroth order single particle Hamiltonian is given by

$$
H_{0}^{\mathrm{cyl}}=-\frac{1}{2 m^{*}}\left[\frac{\partial^{2}}{\partial \rho^{2}}+\frac{1}{\rho} \frac{\partial}{\partial \rho}+\frac{1}{\rho^{2}} \frac{\partial^{2}}{\partial \phi^{2}}+\frac{\partial^{2}}{\partial z^{2}}\right]+\frac{1}{2} m^{*} \omega_{0}^{2} \rho^{2},
$$

where $m^{*}$ is the effective mass of electron. Here, $\hbar$ is taken to be unity. We may consider $\omega_{0}$ as a parameter that controls the confinement of electrons around the $z$ axis. The eigenvalues for $H_{0}^{\text {cyl }}$ are $E_{n m k_{z}}^{0}=\frac{k_{z}^{2}}{2 m^{*}}+\omega_{0}(2 n+|m|+1)$, where $n$ is a non-negative integer and $m$ is an integer, and their eigenfunctions are

$$
\begin{aligned}
\varphi_{n m k_{z} \sigma}(\rho, \phi, z)= & N_{n|m|}\left(m^{*} \omega_{0} \rho^{2}\right)^{\frac{|m|}{2}} L_{n+|m|}^{|m|}\left(m^{*} \omega_{0} \rho^{2}\right) \\
& \times e^{-\frac{m^{*} \omega_{0} \rho^{2}}{2}} e^{i m \phi} e^{i k_{z} z}|\sigma\rangle,
\end{aligned}
$$

where $L_{n+|m|}^{|m|}$ is the associated Laguerre polynomial and $N_{n|m|}$ the normalization constant. We adopt the periodic boundary condition in the $z$ direction, thus, $k_{z}$ is given by $k_{z}=\frac{2 \pi}{L} n_{z}$, where $n_{z}$ is integer and $L$ is the period.

By following the BCS theory, we consider the electron pairing between $\varphi_{n m k_{z} \uparrow}$ and its time-reversal partner $\varphi_{n-m-k_{z} \downarrow}$. Then, the BCS type state vector is given by

$$
\left|\mathrm{BCS}^{\mathrm{cyl}}\right\rangle=\prod_{n m, k_{z}}\left(u_{n m k_{z}}+v_{n m k_{z}} c_{n m k_{z} \uparrow}^{\dagger} c_{n-m-k_{z} \downarrow}^{\dagger}\right)|\mathrm{vac}\rangle
$$

where $c_{n m k_{z} \uparrow}^{\dagger}$ and $c_{n-m-k_{z} \downarrow}^{\dagger}$ denote the creation operators for $\varphi_{n m k_{z} \uparrow}$ and $\varphi_{n-m-k_{z} \downarrow}$, respectively. 
Now we incorporate the Rashba spin-orbit interaction $H_{s o}^{\text {cyl }}$ as a perturbation. By following our previous work [11], it is expected that the pairing of $\varphi_{n m k_{z}}^{a}$ and $\varphi_{n-m-k_{z}}^{b}$ will yield a lower total energy than the pairing in Eq. (5), where $\varphi_{n m k_{z}}^{a}$ and $\varphi_{n m k_{z}}^{b}$ are defined as

$$
\begin{aligned}
\varphi_{n m k_{z}}^{a}(\rho, \phi, z)= & \frac{e^{i\left(m \phi+k_{z} z\right)} R_{n|m+\alpha|}(\rho)}{\sqrt{2 \pi L}} \\
& \times \frac{e^{-\frac{i}{2} \chi^{a}}}{\sqrt{2}}\left(e^{-\frac{i}{2} \phi}|\uparrow\rangle+e^{\frac{i}{2} \phi}|\downarrow\rangle\right) \\
\varphi_{n m k_{z}}^{b}(\rho, \phi, z)= & \frac{e^{i\left(m \phi+k_{z} z\right)} R_{n|m+\alpha|}(\rho)}{\sqrt{2 \pi L}} \\
& \times \frac{e^{-\frac{i}{2} \chi^{b}}}{\sqrt{2}}\left(-e^{-\frac{i}{2} \phi}|\uparrow\rangle+e^{\frac{i}{2} \phi}|\downarrow\rangle\right) .
\end{aligned}
$$

The radial function $R_{n|m+\alpha|}(\rho)$ is defined as follows,

$$
\begin{aligned}
R_{n|m+\alpha|}(\rho) & =\sqrt{\frac{2 m^{*} \omega_{0} n !}{[\Gamma(n+|m+\alpha|+1)]^{3}}}\left(m^{*} \omega_{0} \rho^{2}\right)^{\frac{|m+\alpha|}{2}} \\
& \times L_{n+|m+\alpha|}^{|m+\alpha|}\left(m^{*} \omega_{0} \rho^{2}\right) e^{-\frac{m^{*} \omega_{0} \rho^{2}}{2}}
\end{aligned}
$$

It is normalized as $\int_{0}^{\infty} \rho d \rho R_{n|m+\alpha|}(\rho) R_{n^{\prime}|m+\alpha|}(\rho)=\delta_{n n^{\prime}}$. The value of $\alpha$ will be specified, later.

The phases $\chi^{a}$ and $\chi^{b}$ are introduced to impose the single-valued requirement on $\varphi_{n m k_{z}}^{a}$ and $\varphi_{n m k_{z}}^{b}$. The winding numbers of $\chi^{a}$ and $\chi^{b}$ around the $z$ axis must be odd since the winding number of spin-twisting around $z$ axis is odd since the sum of the two winding numbers must be even [11].

We split $\chi^{a}$ and $\chi^{b}$ as follows;

$$
\chi^{a}=\chi+\chi^{\prime} ; \quad \chi^{b}=\chi-\chi^{\prime}
$$

Then, the part of the phase factor $e^{-i \frac{\chi}{2}}$ in $\varphi_{n m k_{z}}^{a}$ and $\varphi_{n m k_{z}}^{b}$ can be put into the whole system motion part of the wave function in Eq. (1). We only consider the contribution from the phase factors $e^{ \pm i \frac{\chi^{\prime}}{2}}$ for a while.

The spin-densities of the above wave functions are calculated as

$$
\begin{aligned}
& \left(\varphi_{n m k_{z}}^{a}\right)^{*} \hat{s}^{x} \varphi_{n m k_{z}}^{a}=-\left(\varphi_{n m k_{z}}^{b}\right)^{*} \hat{s}^{x} \varphi_{n m k_{z}}^{b}=\frac{R_{n|m+\alpha|}^{2}(\rho) \cos \phi}{2 \pi L} \\
& \left(\varphi_{n m k_{z}}^{a}\right)^{*} \hat{s}^{y} \varphi_{n m k_{z}}^{a}=-\left(\varphi_{n m k_{z}}^{b}\right)^{*} \hat{s}^{y} \varphi_{n m k_{z}}^{b}=\frac{R_{n|m+\alpha|}^{2}(\rho) \sin \phi}{2 \pi L} \\
& \left(\varphi_{n m k_{z}}^{a}\right)^{*} \hat{s}^{z} \varphi_{n m k_{z}}^{a}=\left(\varphi_{n m k_{z}}^{b}\right)^{*} \hat{s}^{z} \varphi_{n m k_{z}}^{b}=0
\end{aligned}
$$


where $\hat{s}^{t}$ is the $t$ component of the spin operator. This shows that $\varphi_{n m k_{z}}^{a}$ has spin-density in the direction $(x, y, z)=(\cos \phi, \sin \phi, 0)$ and $\varphi_{n m k_{z}}^{b}$ has it in the opposite direction.

The current-density of $\varphi_{n m k_{z}}^{t},(t=a, b)$, in the $\phi$ direction is calculated as

$$
\left(\varphi_{n m k_{z}}^{t}\right)^{*} \frac{q}{m^{*} i \rho} \frac{\partial}{\partial \phi} \varphi_{n m k_{z}}^{t}=\frac{q}{m^{*} \rho}\left(m-\frac{1}{2} \frac{\partial \chi^{t}}{\partial \phi}\right) \frac{R_{n|m+\alpha|}^{2}(\rho)}{2 \pi L} .
$$

The current density carried by the $\varphi_{n m k_{z}}^{a}$ and $\varphi_{n-m-k_{z}}^{b}$ pair is, thus, given by

$$
j_{\text {pair }}^{\text {cyl }}=-\frac{q}{2 m^{*} \rho} \frac{\partial\left(\chi^{a}+\chi^{b}\right)}{\partial \phi} \frac{R_{n|m+\alpha|}^{2}(\rho)}{2 \pi L} .
$$

The spin-orbit interaction energy densities for $\varphi_{n m k_{z}}^{a}$ and $\varphi_{n m k_{z}}^{b}$ are calculated as

$$
\begin{aligned}
\left(\varphi_{n m k_{z}}^{a}\right)^{*} H_{s o}^{\mathrm{cyl}} \varphi_{n m k_{z}}^{a} & =\left(\frac{m}{\rho}-\frac{1}{2 \rho} \frac{\partial \chi^{a}}{\partial \phi}\right) \frac{\lambda E_{\mathrm{em}} R_{n|m+\alpha|}^{2}(\rho)}{2 \pi L} \\
\left(\varphi_{n m k_{z}}^{b}\right)^{*} H_{s o}^{\mathrm{cyl}} \varphi_{n m k_{z}}^{b} & =-\left(\frac{m}{\rho}-\frac{1}{2 \rho} \frac{\partial \chi^{b}}{\partial \phi}\right) \frac{\lambda E_{\mathrm{em}} R_{n|m+\alpha|}^{2}(\rho)}{2 \pi L} .
\end{aligned}
$$

The spin-orbit interaction energy density for the pair $\varphi_{n m k_{z}}^{a}$ and $\varphi_{n-m-k_{z}}^{b}$ is given by

$$
h_{s o ; m}^{\mathrm{cyl}}=\lambda E_{\mathrm{em}}\left[\frac{2 m}{\rho}-\frac{1}{2 \rho} \frac{\partial\left(\chi^{a}-\chi^{b}\right)}{\partial \phi}\right] \frac{R_{n|m+\alpha|}^{2}(\rho)}{2 \pi L} .
$$

For simplicity, we consider the case where $\chi^{\prime}$ is an integral multiple of $\phi$. This leads to

$$
\frac{d\left(\chi^{a}-\chi^{b}\right)}{d \phi}=2 \frac{d \chi^{\prime}}{d \phi}=2 \nu
$$

where $\nu$ is an integer. The spin-orbit interaction energy density for the pair $\varphi_{n m k_{z}}^{a}$ and $\varphi_{n-m-k_{z}}^{b}$ is now given by

$$
h_{s o ; m}^{\mathrm{cyl}}=\lambda E_{\mathrm{em}} \frac{2 m-\nu}{\rho} \frac{R_{n|m+\alpha|}^{2}(\rho)}{2 \pi L} .
$$

The kinetic energy density for $\varphi_{n m k_{z}}^{a}$ and $\varphi_{n-m-k_{z}}^{b}$ are equal, given by

$$
\begin{gathered}
\left(\varphi_{n m k_{z}}^{a}\right)^{*} H_{0}^{\mathrm{cyl}} \varphi_{n m k_{z}}^{a}=\frac{R_{n|m+\alpha|}(\rho)}{2 m^{*}}\left[\left(m^{*} \omega_{0}\right)^{2} \rho^{2}\right. \\
\left.-\frac{\partial^{2}}{\partial \rho^{2}}-\frac{1}{\rho} \frac{\partial}{\partial \rho}+\frac{1}{\rho^{2}}\left(\left|m-\frac{\nu}{2}\right|^{2}+\frac{1}{4}\right)+k_{z}^{2}\right] \frac{R_{n|m+\alpha|}(\rho)}{2 \pi L}
\end{gathered}
$$

A sensible choice for $\alpha$ is $\alpha=-\nu / 2$; then, the kinetic energy density for $\varphi_{n m k_{z}}^{a}$ and $\varphi_{n-m-k_{z}}^{b}$ are given by

$$
\left[\omega_{0}\left(2 n+\left|m-\frac{\nu}{2}\right|+1\right)+\frac{1}{8 m^{*} \rho^{2}}+k_{z}^{2}\right] \frac{R_{n\left|m-\frac{\nu}{2}\right|}^{2}(\rho)}{2 \pi L} .
$$


Now we consider the total electronic state. By ansatz use the following BCS type state vector,

$$
\left|\widetilde{\mathrm{BCS}}^{\mathrm{cyl}}\right\rangle=\prod_{n m, k_{z}}\left(u_{n m k_{z}}+v_{n m k_{z}} a_{n m k_{z}}^{\dagger} b_{n-m-k_{z}}^{\dagger}\right)|\mathrm{vac}\rangle
$$

where $a_{n m k_{z}}^{\dagger}$ and $b_{n-m-k_{z}}^{\dagger}$ denote the creation operators for $\varphi_{n m k_{z}}^{a}$ and $\varphi_{n-m-k_{z}}^{b}$, respectively. Note that we keep $u_{n m k_{z}}$ and $v_{n m k_{z}}$ appearing in Eq. (5) for simplicity without optimization. The value of $\nu$ will be optimized.

Let us calculate the energy difference between the ordinary pairing state, $\left|\mathrm{BCS}^{\mathrm{cyl}}\right\rangle$, and spin-twisting-itinerant-motion pairing state, $\left|\widetilde{\mathrm{BCS}}^{\text {cyl }}\right\rangle$ by assuming that the pairing interaction energy is the same for both. The total energy increase by the modification of the pairing states is calculated as

$$
\begin{gathered}
\Delta E^{\mathrm{cyl}} \approx \sum_{n, m, k_{z}} v_{n m k_{z}}^{2}\left[\frac{1}{8 m^{*}} \int_{0}^{\infty} \frac{d \rho}{\rho} R_{n\left|m-\frac{\nu}{2}\right|}^{2}(\rho)-\nu \lambda E_{\mathrm{em}}\right. \\
\left.\times \int_{0}^{\infty} d \rho R_{n\left|m-\frac{\nu}{2}\right|}^{2}(\rho)\right]+\sum_{n, m, k_{z}} v_{n m k_{z}}^{2} \omega_{0}\left(\left|m-\frac{\nu}{2}\right|-|m|\right)
\end{gathered}
$$

It contains terms of different dependences on $\omega_{0}$. If $\omega_{0}$ is larger, the electrons are more localized around the $z$ axis.

In order to estimate the $\omega_{0}$ dependence of terms in $\Delta E^{\text {cyl }}$, we introduce the following function

$$
\tilde{R}_{n\left|m-\frac{\nu}{2}\right|}(X)=X^{\frac{\left|m-\frac{\nu}{2}\right|}{2}} L_{n+\left|m-\frac{\nu}{2}\right|}^{\left|m-\frac{\nu}{2}\right|}(X) e^{-\frac{X}{2}}
$$

which is related to $R_{n\left|m-\frac{\nu}{2}\right|}(\rho)$ as $R_{n\left|m-\frac{\nu}{2}\right|}(\rho)=\sqrt{\frac{2 m^{*} \omega_{0} n !}{\left[\Gamma\left(n+\left|m-\frac{\nu}{2}\right|+1\right)\right]^{3}}} \tilde{R}_{n\left|m-\frac{\nu}{2}\right|}(X)$, where $X=$ $m^{*} \omega_{0} \rho^{2}$.

Using $\tilde{R}_{n\left|m-\frac{\nu}{2}\right|}(X)$, two integrals in Eq. (19) are rewritten as

$$
\int_{0}^{\infty} d \rho R_{n\left|m-\frac{\nu}{2}\right|}^{2}(\rho)=\frac{\sqrt{m^{*} \omega_{0}} n ! \int_{0}^{\infty} \frac{d X}{\sqrt{X}} \tilde{R}_{n\left|m-\frac{\nu}{2}\right|}^{2}(X)}{\left[\Gamma\left(n+\left|m-\frac{\nu}{2}\right|+1\right)\right]^{3}}
$$

and

$$
\int_{0}^{\infty} \frac{d \rho}{\rho} R_{n\left|m-\frac{\nu}{2}\right|}^{2}(\rho)=\frac{m^{*} \omega_{0} n ! \int_{0}^{\infty} \frac{d X}{X} \tilde{R}_{n\left|m-\frac{\nu}{2}\right|}^{2}(X)}{\left[\Gamma\left(n+\left|m-\frac{\nu}{2}\right|+1\right)\right]^{3}} .
$$

The value of $\nu$ should be optimized to minimize $\Delta E^{\mathrm{cyl}}$ under the condition, $\nu \lambda E_{\mathrm{em}}>0$. 
The above integrals indicate that $\Delta E^{\mathrm{cyl}}$ becomes negative for a sufficiently small value of $\omega_{0}$ since the positive terms scale as $\omega_{0}$ and the negative one scales as $\sqrt{\omega_{0}}$. This means that $\varphi_{n m k_{z}}^{a}$ and $\varphi_{n-m-k_{z}}^{b}$ pairing becomes more stable than the original BCS type pairing $\varphi_{n m k_{z} \uparrow}$ and $\varphi_{n-m-k_{z} \downarrow}^{b}$ if the spin-twisting circular current region is sufficiently spread.

We may regard that the particle number $N$ projection from $\left|\widetilde{\mathrm{BCS}}^{\text {cyl }}\right\rangle$ gives the wave function of the system given in Eq. (1). If $\nu$ is even, the winding number of $\chi^{\prime}$ around the $z$ axis is even; then, the winding number of $\chi$ around the $z$ axis must be odd. This means that a nontrivial whole system motion is generated by the phase factor $e^{-\frac{i}{2} \sum_{\alpha=1}^{N} \chi\left(\mathbf{r}^{(\alpha)}\right)}$.

Now, we add a uniform magnetic field in the $z$ direction. In order to include the magnetic field $B$, we adopt the symmetric vector potential given by $\mathbf{A}^{\mathrm{em}}=\left(-\frac{B}{2} y, \frac{B}{2} x, 0\right)$.

The free electron with effective mass $m^{*}$ in the magnetic field is given by

$$
\begin{aligned}
H_{\text {mag }}^{\text {cyl }}= & -\frac{1}{2 m^{*}}\left[\frac{\partial^{2}}{\partial \rho^{2}}+\frac{1}{\rho} \frac{\partial}{\partial \rho}+\frac{1}{\rho^{2}} \frac{\partial^{2}}{\partial \phi^{2}}+\frac{\partial^{2}}{\partial z^{2}}\right] \\
& +\frac{1}{8} m^{*} \omega_{c}^{2} \rho^{2}+\frac{\omega_{c}}{2 i} \frac{\partial}{\partial \phi}
\end{aligned}
$$

Thus, the magnetic field generates the quadratic confining potential with $\omega_{0}=\frac{1}{2} \omega_{c}=$ $\frac{1}{2} \sqrt{\frac{e B}{m^{*} c}}$, where $\omega_{c}$ is the cyclotron angular frequency.

We add the pairing interaction, and take the term $\frac{\omega_{c}}{2 i} \frac{\partial}{\partial \phi}$ as a perturbation. Then, the zeroth order wave function is given as the BCS-type state vector $\left|\widetilde{\mathrm{BCS}}^{\text {cyl }}\right\rangle$ in Eq. (18) with replacing $\omega_{0}$ by $\frac{1}{2} \omega_{c}=\frac{1}{2} \sqrt{\frac{e B}{m^{*} c}}$. We also add the Rashba interaction as a perturbation. The energy increase by the perturbations is given as a formula in Eq. (19) with an additional contribution from the term $\frac{\omega_{c}}{2 i} \frac{\partial}{\partial \phi}$. The added term gives a contribution that scales as $\omega_{c}$. Due to the Rashba interaction contribution that scales as $\sqrt{\omega_{c}}$, the energy increase becomes negative in a sufficiently weak magnetic field, thus, the spin-twisting itinerant motion of electrons is stabilized.

When $\nu$ is an even number, nontrivial $\chi$ is realized since the winding number of $\chi$ around the $z$ axis must be odd. The nontrivial $\chi$ generates a loop current forced to flow by the singlevalued requirement of the wave function. Since the central position of the spin-twisting is arbitrary, the spin-twisting itinerant motion of electrons may occur at different places by shifting central positions, simultaneously. If this happens, it gives rise to a distribution of stable eddy currents in an analogous manner observed in the Abrikosov vortex system [27]. A macroscopic supercurrent will be generated as a collection of them. The present work 
suggests that upon an application of an external magnetic field, a distribution of stable eddy currents generated by nontrivial $\chi$ appears and gives rise to the Meissner effect.

In conclusion, we have shown that the new supercurrent generation mechanism by the spin-twisting itinerant motion of electrons is applicable to the BCS superconductor by adding the Rashba spin-orbit interaction to the BCS theory. It is effective even for a weak Rashba interaction if the magnetic field is sufficiently weak. This supercurrent generation mechanism conserves the particle number. The BCS-type state vector is used only to facilitate calculations involving the electron pairing.

[1] J. Bardeen, L. N. Cooper, and J. R. Schrieffer, Phys. Rev. 108, 1175 (1957).

[2] V. L. Ginzburg and L. D. Landau, Zh. Exsp. Teor. Fiz. 20, 1064 (1950).

[3] L. P. Gor'kov, Sov. Phys. JETP 9, 1364 (1959).

[4] B. D. Josephson, Phys. Lett. 1, 251 (1962).

[5] J. G. Bednorz and K. A. Müller, Z. Phys. B64, 189 (1986).

[6] V. J. Emery and S. A. Kivelson, Nature 374, 434 (1995).

[7] H. Koizumi, J. Supercond. Nov. Magn. 24, 1997 (2011).

[8] R. Hidekata and H. Koizumi, J. Supercond. Nov. Magn. 24, 2253 (2011).

[9] H. Koizumi, R. Hidekata, A. Okazaki, and M. Tachiki, J Supercond Nov Magn 27, 121 (2014).

[10] H. Koizumi, A. Okazaki, M. Abou Ghantous, and M. Tachiki, J. Supercond. Nov. Magn. 27, 2435 (2014).

[11] H. Koizumi and M. Tachiki, J. Supercond. Nov. Magn. pp. DOI 10.1007/s10948-014-2877-5 (2014).

[12] E. Schrödinger, Ann. Physik 79, 361 (1926).

[13] H. C. Longuet-Higgins, U. Öpik, M. H. L. Pryce, and R. A. Sack, Proc. Roy. Soc. London Ser. A 244, 1 (1958).

[14] M. D. Fiske, Rev. Mod. Phys. 36, 221 (1963).

[15] I. K. Yanson, V. M. Svistunov, and I. M. Dmitrenko, J. Exptl. Theoret. Phys. (U.S.S.R.) 48, 976 (1965).

[16] I. Giavever, Phys. Rev. Lett. 14, 904 (1965).

[17] G. Rickayzen, Theory of superconductivity (Interscience, New York, 1965). 
[18] J. R. Waldram, Rep. Prog. Phys. 39, 751 (1976).

[19] R. P. Feynman, R. Leighton, and M. Sands, Lectures on physics, vol. 3 (Addison-wesley, Reading MA, 1965).

[20] C. Kittel, Introductio to Solid State Physics (Wiley, USA, 1986), 6th ed.

[21] M. Tinkham, Introduction to superconductivity (MacGraw-Hill, USA, 1996), 2nd ed.

[22] P. W. Anderson, Phys. Rev. 112, 1900 (1958).

[23] Y. Nambu, Phys. Rev. 117, 648 (1960).

[24] G. C. Wick, A. S. Wightman, and E. P. Wigner, Phys. Rev. D 1, 3267 (1970).

[25] R. Peierls, Contemporary Phys. 33, 221 (1992).

[26] E. I. Rashba, Sov. Phys. Solid State 2, 1109 (1960).

[27] A. A. Abrikosov, Sov. Phys. JETP 5, 1174 (1957). 